\title{
Control of Synchronization and Stability for Nonlinear Complex Dynamical Networks with Different Dimensional Similar Nodes and Coupling Time-Varying Delay
}

\author{
Luo Yi-ping, Luo Xin, Deng Fei, and Hu Jun-qiang \\ Hunan Institute of Engineering, Xiangtan, Hunan 411104, China \\ Correspondence should be addressed to Luo Xin; 97646021@qq.com
}

Received 10 January 2015; Revised 25 March 2015; Accepted 30 March 2015

Academic Editor: Xinggang Yan

Copyright (c) 2015 Luo Yi-ping et al. This is an open access article distributed under the Creative Commons Attribution License, which permits unrestricted use, distribution, and reproduction in any medium, provided the original work is properly cited.

\begin{abstract}
This paper discusses the stability and synchronization for the nonlinear coupled complex networks with different dimensional nodes, and the external coupling satisfies the condition of dissipation. The definition of synchronization of the complex dynamical networks is proposed as the manifold. By Lyapunov stability theorem, the decentralized controllers with similar parameters are designed to synchronize such dynamical networks asymptotically in which the characteristics are variable delayed. Finally, a numerical example is given to illustrate the effectiveness of the designed method.
\end{abstract}

\section{Introduction}

Extensively existing in various phenomena of all kinds of areas in the world, such as social network and World Wide Web (WWW), complex dynamical networks have received more and more attention in recent years [1, 2]. A lot of researchers have analyzed coupling complex dynamical networks and got abundant results [3-7]. Representative phenomenon in complex dynamical networks is synchronization among all dynamical nodes. So in the past few years, synchronization is the interesting subject for researchers [810]. As a result, widespread and varied criteria for stability and synchronization in dynamical network have been derived [11-14]. For instance, [13] discussed the adaptive pinning synchronization in complex networks with nondelay and variable delay coupling. Reference [14] designed controllers with synchronization conditions to achieve the synchronization of nonlinear coupled dynamic complex networks with unanimous delay. Anyway the existing works on this topic normally focus on the dynamical complex network with same dimensions of nodes.

However, attributes of individual node might be different. Such as the Super Smart Grid, every user as a node possesses similar but discrepant equipment, and every kind of equipment of user is one dimension of node. By using different dimensions of node to express different attributes of user, we can define the Super Smart Grid as complex networks with different dimensional similar nodes.

Though [15] mentioned that one synchronization scheme is applicable to the complex networks in which the nodes contain different dimensions, both the nodes and coupling of nodes are linear. In reality of engineering, the nonlinear coupling might be more complicated than the linear system. Recently, [16] also researched coupled complex dynamical networks with different dimensions nodes, which discussed the asymptotic synchronization of this network by decentralized dynamical compensation controllers without mentioning time delay. As we all know, time delay widely exists in various phenomena of nature, engineering networks, biological system, and human social activities. Generally speaking, time delay is inevitable because the information spread through a complex network that is characterized by limited speed of long distance signal transmission. Furthermore, in some real situations delay is varied, which is called time-varying delay.

All of the above discussions are to construct the nonlinear complex dynamical networks with different dimensional similar nodes and coupling time-varying delay. In this paper, we assumed that the dimension of individual node in this coupled complex dynamical network is different. Since nodes have some similar behaviors and state connections, we define 
the synchronization of the dynamical networks as the manifold. Considering the time-varying delay in this network, the decentralized control strategies are designed to achieve the stability and synchronization asymptotically for similar nodes of complex dynamical networks. In the end, numerical examples are given to demonstrate the effectiveness of our proposed results. Finally, Section 5 presents conclusions.

\section{Formulation of the Problem and Preliminaries}

Here we consider a complex dynamical network which has similar nodes with different dimensions and coupling timevarying delay:

$$
\begin{aligned}
\dot{x}_{i}= & E_{i} x_{i} \\
& +\sum_{j=1, j \neq i}^{N} c_{i j}\left(h_{i j}\left(x_{j}(t-\tau(t))\right)-h_{i i}\left(x_{i}(t-\tau(t))\right)\right) \\
& +F_{i} u_{i},
\end{aligned}
$$

where the $i$ th node satisfies, $i=1,2, \ldots, N, x_{i} \in R^{n_{i}}$ and $x_{i}=\left(x_{i 1}, x_{i 2}, \ldots, x_{i n_{i}}\right)^{T}$ and $u_{i} \in R^{m \times n_{i}}$ is the control input of node $i$. Respectively, $E_{i} \in R^{n_{i} \times n_{i}}, F_{i} \in R^{n_{i} \times m}$ both are known as constant matrices. The sufficiently smooth $h_{i j}$ are the nonlinear vector fields, defined as $R^{n_{j}} \rightarrow R^{n_{i}}(i, j=$ $1,2, \ldots, N) ; c_{i j} \in R$ are the outer coupling configuration parameters. $c_{i j}$ shows the coupling strength and topology of the dynamical network and satisfies $c_{i i}=-\sum_{j=1, j \neq i}^{N} c_{i j}$. And $c_{i j} \neq 0$ if there is a connection between node $i$ and node $j(i \neq j)$; otherwise $c_{i j}=0$. The diagonal elements of matrix are defined as $C=\left(c_{i j}\right)_{N \times N}$.

Assumption 1. Consider network (1) of $N$ different dimensional nodes. There exist $N$ matrixes $S_{i} \in R^{n_{1} \times n_{i}}\left(S_{i} \neq 0\right)$, $B_{i} \in R^{m \times n_{i}}$, and $u_{i}=B_{i} x_{i}+A_{i} v_{i}$ conforming to

$$
\begin{aligned}
S_{i}\left(E_{i}+F_{i} B_{i}\right) & =\left(E_{1}+F_{1} A_{1}\right) S_{i}, \\
S_{i} F_{i} & =F_{1},
\end{aligned}
$$

where $i=1, \ldots, N$.

Assumption 2. Under Assumption 1 conditions, there exist $H_{i} \in R^{n_{1} \times n_{1}}$ and $i=1, \ldots, N$ satisfying

$$
S_{i} h_{i j}\left(x_{i}(t-\tau(t))\right)=H_{i} S_{j} x_{j}(t-\tau(t)) .
$$

Remark 3. Notice every node of the complex dynamical network has its own dimensions, and there is no certain connection for the dimension among different nodes. Assumption 1 shows $N$ matrixes $E_{i}+F_{i} B_{i}$ have some same eigenvalues. It further means that the state of different nodes contains similar behaviors.

Lemma 4 (see [17]). For any vectors $x, y \in R^{n \times n}$ and positive definite matrix $W \in R^{n \times n}$, the following matrix inequity holds: $2 x^{T} y \leq x^{T} W x+y^{T} W^{-1} y$.

\section{Synchronization for Complex Network by Decentralized Controllers}

In this section, based on Assumptions 1-2 and Lemma 4, at first we propose the definition of synchronization manifold and then synthesise the decentralized dynamical compensation controllers to synchronize the complex network asymptotically.

Definition 5. A complex dynamical network is said to achieve the asymptotical synchronization if

$$
S_{1} x_{1}=S_{2} x_{2}=\cdots=S_{i} x_{i}=s(t), \quad \text { as } t \longrightarrow \infty
$$

where $s(t) \in R^{n_{1}}$ is a solution of targeted state, satisfying

$$
\dot{s}(t)=F_{1} s(t) .
$$

For our synchronization scheme, we define the error vectors as

$$
e_{i}=S_{i} x_{i}-s(t)
$$

From (5) and (6), Assumptions 1 and 2, the dynamical error equation is given as

$$
\begin{aligned}
\dot{e} & =S_{i} \dot{x}_{i}-\dot{s}(t)=S_{i}\left[E_{i} x_{i}+F_{i} u_{i}\right. \\
& \left.+\sum_{j=1, j \neq i}^{N} c_{i j}\left(h_{i j}\left(x_{j}(t-\tau(t))\right)-h_{i i}\left(x_{i}(t-\tau(t))\right)\right)\right] \\
& -E_{1} s(t)=S_{i}\left[\left(E_{i}+F_{i} B_{i}\right) x_{i}+F_{i} A_{i} v_{i}\right. \\
& \left.+\sum_{j=1}^{N} c_{i j} h_{i j}\left(x_{j}(t-\tau(t))\right)\right]-E_{1} s(t)-\sum_{j=1}^{N} c_{i j} H_{i} s(t) \\
& =\left(E_{i}+F_{1} B_{1}\right) S_{i} x_{i}+F_{1} A_{1} v_{i} \\
& +\sum_{j=1}^{N} c_{i j} H_{i} S_{j} x_{j}(t-\tau(t))-E_{1} s(t) \\
& -\sum_{j=1}^{N} c_{i j} H_{i} s(t-\tau(t))=E_{1} e_{i}+F_{1} B_{1} S_{i} x_{i}+F_{1} A_{1} v_{1} \\
& +\sum_{j=1}^{N} c_{i j} H_{i} e_{j}(t-\tau(t)),
\end{aligned}
$$

where $\left\|H_{i}\right\| \in R^{n_{1} \times n_{1}}(i=1, \ldots, N)$ are norm-bounded, which means there is a constant $M$ satisfying

$$
\left\|H_{i}\right\| \leq M .
$$


Theorem 6. In this section, one decentralized dynamical compensation controller is designed to achieve our synchronization as mentioned before, which is

$$
\begin{aligned}
& u_{i}=B_{i} x_{i}+A_{i} v_{i}, \\
& v_{i}=d_{i} A_{1}^{-1} F_{1 R}^{-1} e_{i}-A_{1}^{-1} B_{1} S_{i} x_{i},
\end{aligned}
$$

where $i=1, \ldots, N$, constant $k_{i}>0 . F_{1 R}^{-1}$ is a right inverse matrix for $F_{1}$ and satisfies $F_{1} F_{1 R}^{-1}=I_{n_{1}}$, which generally means $F_{1}$ has full row rank.

Here from (9), error dynamical systems (7) become the following form:

$$
\dot{e}_{i}=E_{1} e_{i}+d_{i} e_{i}+\sum_{j=1}^{N} c_{i j} H_{i} e_{j}(t-\tau(t)),
$$

$$
(i=1, \ldots, N)
$$

Select $\widehat{c}=\max _{1<i<N}\left|c_{i j}\right|$ and $\delta=\max \operatorname{eig}\left(\left(E_{1}^{T}+E_{1}\right) / 2\right)$. If there exists a constant $\gamma$ satisfying

$$
\begin{aligned}
& \left(\delta+d_{i}+\frac{\widehat{c} M N}{2}+\gamma\right)<0, \\
& \gamma>\frac{\widehat{c} M N}{2}>0,
\end{aligned}
$$

then the error dynamical systems will achieve asymptotical synchronization.

Proof. First, we select the following Lyapunov function candidate:

$$
V(t)=\sum_{i=1}^{N} \frac{e_{i}^{T} e_{i}}{2}
$$

The derivative of $V(t)$ along the trajectories with error dynamical systems is

$$
\begin{aligned}
\dot{V}(t)= & \sum_{i=1}^{N}\left(\frac{\dot{e}_{i}^{T} e_{i}}{2}+\frac{e_{i}^{T} \dot{e}_{i}}{2}\right) \\
= & \sum_{i=1}^{N}\left[\frac{e_{i}^{T}\left(F_{1}^{T}+F_{1}\right) e_{i}}{2}+d_{i} e_{i}^{T} e_{i}\right] \\
& +\sum_{i=1}^{N} \sum_{j=1}^{N} c_{i j} e_{i}^{T} H_{i} e_{j}(t-\tau(t)) \\
\leq & (\delta+d) e_{i}^{T} e_{i}+\sum_{i=1}^{N} \sum_{j=1}^{N}\left|c_{i j}\right|\left\|H_{i}\right\|\left|e_{i}^{T} e_{j}(t-\tau(t))\right|,
\end{aligned}
$$

where $d=\max _{1<i<N}\left|d_{i}\right|$. By Lemma 4, (11), (12), and the parameters we set before, we derive the following inequation:

$$
\begin{aligned}
\dot{V}(t) \leq & \left(\delta+d+\frac{\widehat{c} M N}{2}\right) \sum_{i=1}^{N} e_{i}^{T} e_{i} \\
& +\frac{\widehat{c} M N}{2} \sum_{j=1}^{N} e_{j}^{T}(t-\tau(t)) e_{j}(t-\tau(t)) \\
\leq & -\gamma \sum_{i=1}^{N} e_{i}^{T} e_{i} \\
& +\frac{\widehat{c} M N}{2} \sum_{j=1}^{N} e_{j}^{T}(t-\tau(t)) e_{j}(t-\tau(t)) .
\end{aligned}
$$

If there exists a function $g(\varepsilon)$ satisfying (12), where

$$
g(\varepsilon)=\varepsilon-\gamma+\frac{\widehat{c} M N}{2} \exp (\varepsilon \tau)
$$

we derive the following results:

$$
\begin{gathered}
g(0)<0, \\
g(\infty)>0, \\
\dot{g}(\varepsilon)>0 .
\end{gathered}
$$

Then from 0 to $\infty$, we can find $\varepsilon>0$ to suit

$$
g(\varepsilon)=\varepsilon-\gamma+\frac{\widehat{c} M N}{2} \exp (\varepsilon \tau)=0
$$

Set

$$
\begin{aligned}
M_{0} & =\sup _{-\tau \leq s \leq 0} V(s), \\
W(t) & =\exp (\varepsilon \tau) V(t), \quad t \geq 0, \\
Q(t) & =W(t)-h M_{0}, \quad h>1 .
\end{aligned}
$$

Hence based on Lyapunov lemma, it is available to make the state of dynamics systems achieve synchronization asymptotically under manifold (4) if we can prove $Q(t)<0, t \in$ $(-\tau, \infty)$.

Firstly, because of $Q(t)=W(t)-h M_{0}$, then $Q(t)<$ $0, t \in(-\tau, 0)$. Secondly, the target is to prove the following inequation:

$$
Q(t)<0, \quad t \in(0, \infty) .
$$

If there exists $t_{0} \in(0, \infty)$, which satisfies

$$
\begin{aligned}
& Q\left(t_{0}\right)=0, \\
& \dot{Q}\left(t_{0}\right)>0, \\
& Q(t)<0, \quad-\tau \leq t \leq t_{0},
\end{aligned}
$$


the time derivative of $Q\left(t_{0}\right)$ is

$$
\begin{aligned}
\dot{Q}\left(t_{0}\right)= & \varepsilon W\left(t_{0}\right)+\exp \left(\varepsilon t_{0}\right) \dot{V}\left(t_{0}\right) \\
\leq & \varepsilon W\left(t_{0}\right)-\gamma \exp \left(\varepsilon t_{0}\right) V\left(t_{0}\right) \\
& +\frac{\widehat{c} M N}{2} \exp \left(\varepsilon t_{0}\right) V\left(t_{0}-\tau(t)\right) \\
\leq & (\varepsilon-\gamma) W\left(t_{0}\right) \\
& +\frac{\widehat{c} M N}{2} \exp (\varepsilon \tau) W\left(t_{0}-\tau(t)\right) \\
< & \left(\varepsilon-\gamma+\frac{\widehat{c} M N}{2} \exp (\varepsilon \tau)\right) h M_{0} .
\end{aligned}
$$

It is clear that there is a contradiction between (22) and (24), so (19) is positive, and according to hypothesis (19), we set $h \rightarrow 1$; then

$$
V(t)<\exp (-\varepsilon t) M_{0}
$$

The proving for Theorem 6 ends.

Remark 7. Compared with other similar published results, this paper discussed the complex dynamical networks with time-varying delay, with designing different controllers. By defining a new function $V$, we find a new way to prove relevant problems and get some results for complex dynamical networks with time-varying delay. Under the research conditions of similar literatures having no time delay, it is available to accept that the method in this paper can simplify the process and save cost of control while we discuss one kind of complex dynamical network without uncertain parts.

\section{Numerical Examples}

The following dynamical error equation illustrates the theoretical effectiveness of our proposed synchronization themes derived in Section 3. To put it simply, we choose a tennode network, in which the first node has 2 dimensions, the second node has 3 dimensions, and the rest of them have 4 dimensions. Consider

$$
\dot{e}_{i}=E_{1} e_{i}+d_{i} e_{i}+\sum_{j=1}^{N} c_{i j} H_{i} e_{j}(t-\tau(t)),
$$

$$
(i=1, \ldots, N)
$$

where we choose the upper bound about time-varying delay as $\tau=0.1$, and without loss of generality, the parameters for dynamical error equation are chosen as follows:

$$
\begin{aligned}
& E_{1}=\left(\begin{array}{cc}
-2 & 5 \\
0 & 3
\end{array}\right), \\
& E_{2}=\left(\begin{array}{ccc}
-2 & 2 & 3 \\
0 & 1 & 2 \\
0 & 0 & 1
\end{array}\right) \\
& E_{3}=\cdots=E_{10}=\left(\begin{array}{cccc}
-2 & 2 & 3 & 5 \\
0 & 1 & 2 & -1 \\
0 & 0 & 1 & 0 \\
0 & 0 & 0 & \text { rand }+2
\end{array}\right) \text {, } \\
& B_{1}=\left(\begin{array}{ll}
1 & 0 \\
0 & 1
\end{array}\right) \text {, } \\
& B_{2}=\left(\begin{array}{ccc}
1 & -1 & -2 \\
0 & 1 & -1
\end{array}\right) \text {, } \\
& B_{i}=\left(\begin{array}{cccc}
1 & -1 & -2 & -3 \\
0 & 1 & -1 & 1
\end{array}\right) \text {, } \\
& A_{j}=\left(\begin{array}{cc}
-1 & 0 \\
3 & 2
\end{array}\right) \text {, }
\end{aligned}
$$

where $i=3,4, \ldots, 10 ; j=1,2, \ldots, 10$. Consider

$$
\begin{aligned}
& S_{1}=\left(\begin{array}{ll}
1 & 0 \\
0 & 1
\end{array}\right), \\
& S_{2}=\left(\begin{array}{lll}
1 & 3 & 0 \\
0 & 1 & 0
\end{array}\right), \\
& S_{3}=\cdots=S_{10}=\left(\begin{array}{llll}
1 & 3 & 0 & 0 \\
0 & 1 & 0 & 0
\end{array}\right) .
\end{aligned}
$$

Consider the connection type for dynamical network, so we choose the outer coupling matrix as

C

$$
=\left(\begin{array}{cccccccccc}
-1 & 0 & 0 & 0 & 1 & 0 & 0 & 0 & 0 & 0 \\
1 & -3 & -1 & 1 & 0 & 0 & 1 & 0 & 0 & 1 \\
0 & 1 & -5 & 1 & 1 & 1 & 1 & -1 & 0 & 1 \\
1 & 1 & 1 & -8 & 1 & 1 & 0 & 1 & 1 & 1 \\
0 & 0 & 1 & 1 & -3 & 0 & 0 & 1 & 0 & 0 \\
0 & 1 & 1 & 1 & 0 & -6 & 1 & 1 & 1 & 0 \\
0 & 1 & 0 & 0 & 0 & 1 & -3 & 0 & 1 & 0 \\
0 & 0 & 0 & 1 & 1 & 0 & 0 & -2 & 0 & 0 \\
0 & 0 & 0 & 0 & 0 & 0 & 1 & 0 & -1 & 0 \\
0 & 1 & 0 & 0 & 0 & 0 & 0 & 0 & 0 & -1
\end{array}\right)_{10 \times 10}
$$




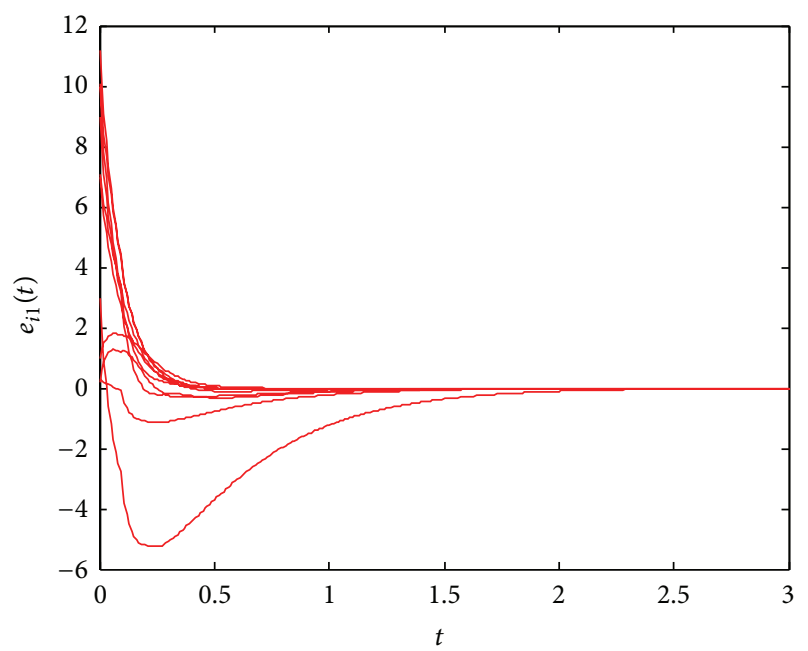

FIGURE 1: State of error for the first dimension of nodes.

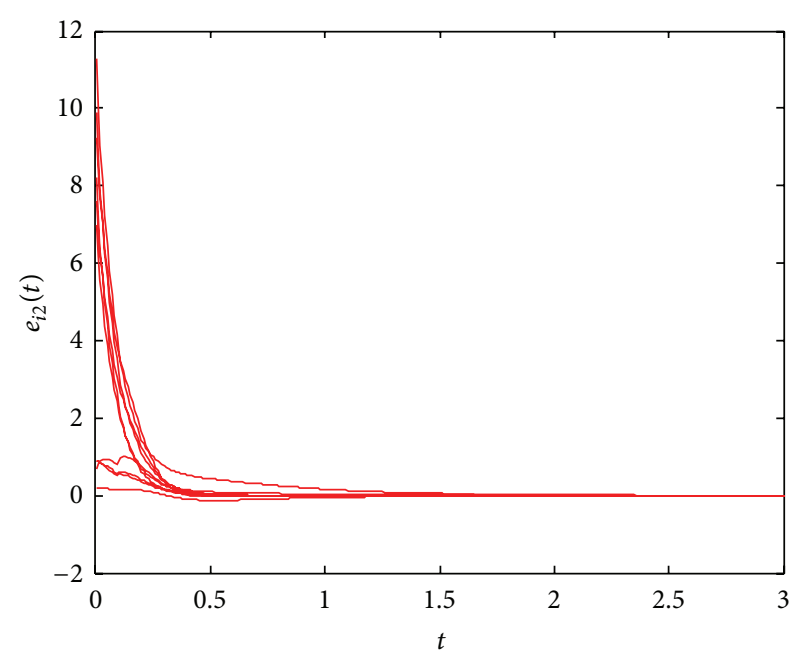

FIGURE 2: State of error for the second dimension of nodes.

In this example, initial conditions of each node are chosen as

$$
\begin{aligned}
& x_{1}^{0}=\left[\begin{array}{ll}
1 & 2
\end{array}\right], \\
& x_{2}^{0}=\left[\begin{array}{lll}
3 & 4 & 5
\end{array}\right], \\
& x_{3}^{0}=\left[\begin{array}{llll}
-0.5 & 2 & 1 & 4
\end{array}\right], \\
& x_{4}^{0}=\left[\begin{array}{llll}
1 & 1.2 & 0 & 1
\end{array}\right], \\
& x_{5}^{0}=\left[\begin{array}{llll}
-5 & 4 & 3 & 5
\end{array}\right], \\
& x_{6}^{0}=\left[\begin{array}{llll}
2 & -4 & 2 & 1
\end{array}\right], \\
& x_{7}^{0}=\left[\begin{array}{llll}
-2 & 3 & -1 & 1
\end{array}\right], \\
& x_{8}^{0}=\left[\begin{array}{llll}
3 & 1 & 1 & 0
\end{array}\right], \\
& x_{9}^{0}=\left[\begin{array}{llll}
0 & 5 & 3 & 2
\end{array}\right], \\
& x_{10}^{0}=\left[\begin{array}{llll}
2 & 1 & -0.5 & 2
\end{array}\right] .
\end{aligned}
$$

Besides, according to Theorem 6 and the assumptions we proposed before, $\left\|H_{i}\right\|<1=M$, and $\delta=4$. Also we are able to find out $d_{i}<-10$ after calculating these parameters. The results are shown in Figures 1 and 2, which exhibit that the dynamical networks achieve synchronization asymptotically.

\section{Conclusions}

In this paper, for the coupling time-varying delay complex networks with different dimensional similar nodes, the decentralized controllers are designed to synchronize such networks. According to the results of numerical example, just if the nodes contain similar behaviors, by Lyapunov stability theorem, we can find decentralized controllers with similar parameters to verify that our stability and synchronization control theme in this paper is effective.

\section{Conflict of Interests}

The authors declare that there is no conflict of interests regarding the publication of this paper.

\section{Acknowledgment}

This work was supported by Natural Science Foundation of China under Grant no. 11372107 and no. 61174211.

\section{References}

[1] H. Liu, J. Chen, J.-A. Lu, and M. Cao, "Generalized synchronization in complex dynamical networks via adaptive couplings," Physica A, vol. 389, no. 8, pp. 1759-1770, 2010.

[2] X. F. Wang and G. Chen, "Complex networks: small-world, scale-free and beyond," IEEE Circuits and Systems Magazine, vol. 3, no. 1, pp. 6-20, 2003.

[3] X. Wang and J. Z. Huang, "Editorial: uncertainty in learning from big data," Fuzzy Sets and Systems, vol. 258, pp. 1-4, 2015.

[4] C. Vitolo, Y. Elkhatib, D. Reusser, C. J. Macleod, and W. Buytaert, "Web technologies for environmental Big Data," Environmental Modelling \& Software, vol. 63, pp. 185-198, 2015.

[5] W. J. Mallon, "Big data," Journal of Shoulder and Elbow Surgery, vol. 22, no. 9, p. 1153, 2013.

[6] K. Kambatla, G. Kollias, V. Kumar, and A. Grama, "Trends in big data analytics," Journal of Parallel and Distributed Computing, vol. 74, no. 7, pp. 2561-2573, 2014.

[7] C. Zheng, M. Sun, Y. Tao, and L. Tian, "Adaptive-impulsive control for generalized projective synchronization between two complex networks with time delay," in Proceedings of the Chinese Control and Decision Conference (CCDC '10), pp. 3574-3578, Xuzhou, China, May 2010.

[8] H. R. Karimi, "Robust synchronization and fault detection of uncertain master-slave systems with mixed time-varying delays and nonlinear perturbations," International Journal of Control, Automation and Systems, vol. 9, no. 4, pp. 671-680, 2011.

[9] D. H. Ji, J. H. Park, W. J. Yoo, S. C. Won, and S. M. Lee, "Synchronization criterion for Lur'e type complex dynamical networks with time-varying delay," Physics Letters A, vol. 374, no. 10 , pp. $1218-1227,2010$. 
[10] N. Li, Y. Zhang, J. Hu, and Z. Nie, "Synchronization for general complex dynamical networks with sampled-data," Neurocomputing, vol. 74, no. 5, pp. 805-811, 2011.

[11] T. Chen, X. Liu, and W. Lu, "Pinning complex networks by a single controller," IEEE Transactions on Circuits and Systems I: Regular Papers, vol. 54, no. 6, pp. 1317-1326, 2007.

[12] X. F. Wang and G. Chen, "Pinning control of scale-free dynamical networks," Physica A: Statistical Mechanics and Its Applications, vol. 310, no. 3-4, pp. 521-531, 2002.

[13] Y. Liang, X. Wang, and J. Eustace, "Adaptive synchronization in complex networks with non-delay and variable delay couplings via pinning control," Neurocomputing, vol. 123, pp. 292-298, 2014.

[14] B. Zhuo, "Pinning synchronization of a class of complex dynamical network with doupling delay," International Journal of Nonlinear Science, vol. 9, no. 2, pp. 207-212, 2010.

[15] Y. H. Wang, Y. Q. Fan, Q. Y. Wang, and Y. Zhang, "Stabilization and synchronization of complex dynamical networks with different dynamics of nodes via decentralized controllers," IEEE Transactions on Circuits and Systems I: Regular Papers, vol. 59, no. 8, pp. 1786-1795, 2012.

[16] L. Zhang, Y. Wang, and Q. Wang, "Synchronization for nonlinearly coupled complex dynamical networks with different dimensional nodes," in Proceedings of the 26th Chinese Control and Decision Conference (CCDC '14), pp. 3632-3637, IEEE, Changsha, China, May- June 2014.

[17] J. Wu and L. Jiao, "Synchronization in complex delayed dynamical networks with nonsymmetric coupling," Physica A, vol. 386, no. 1, pp. 513-530, 2007. 


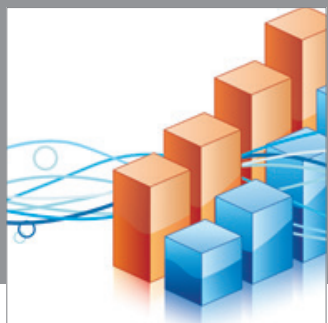

Advances in

Operations Research

mansans

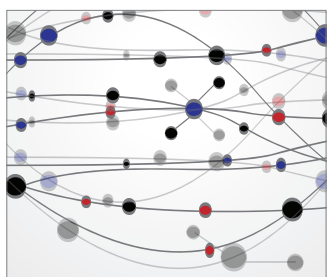

The Scientific World Journal
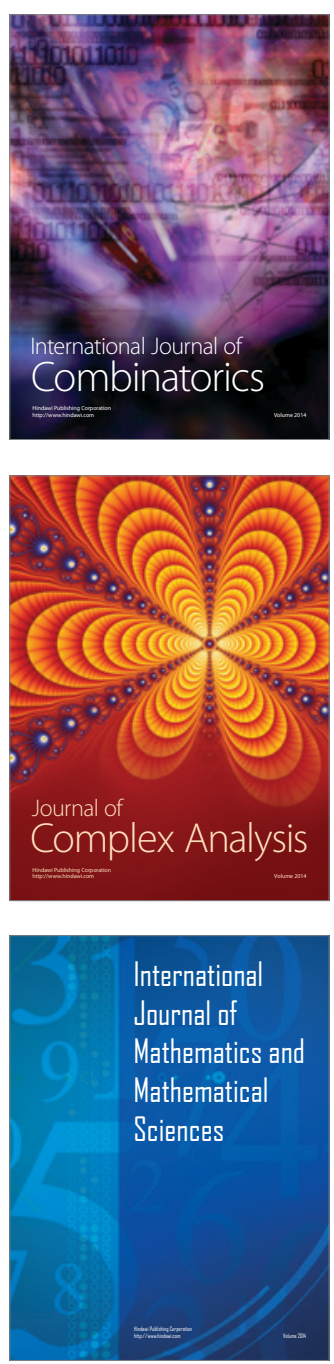
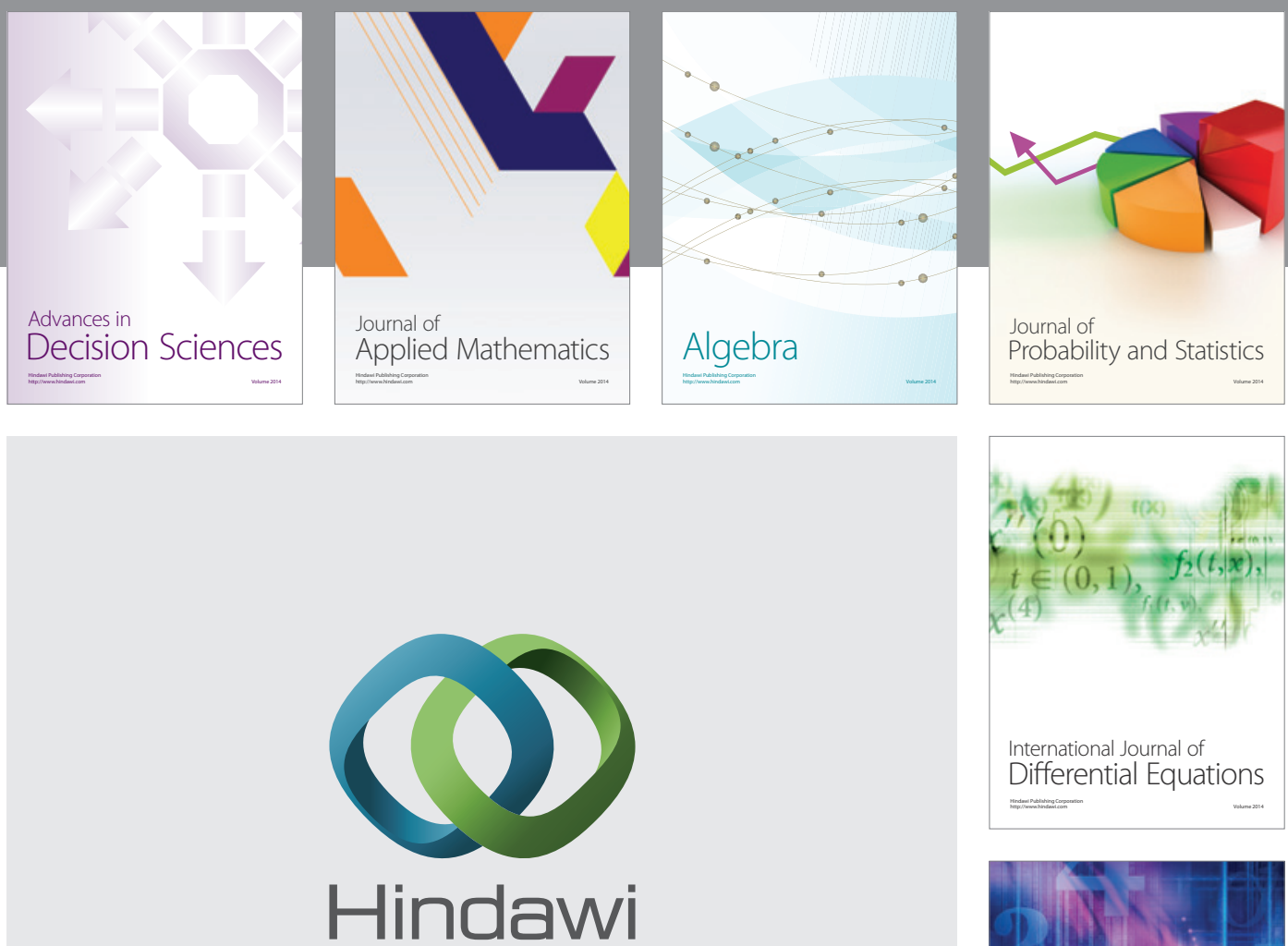

Submit your manuscripts at http://www.hindawi.com
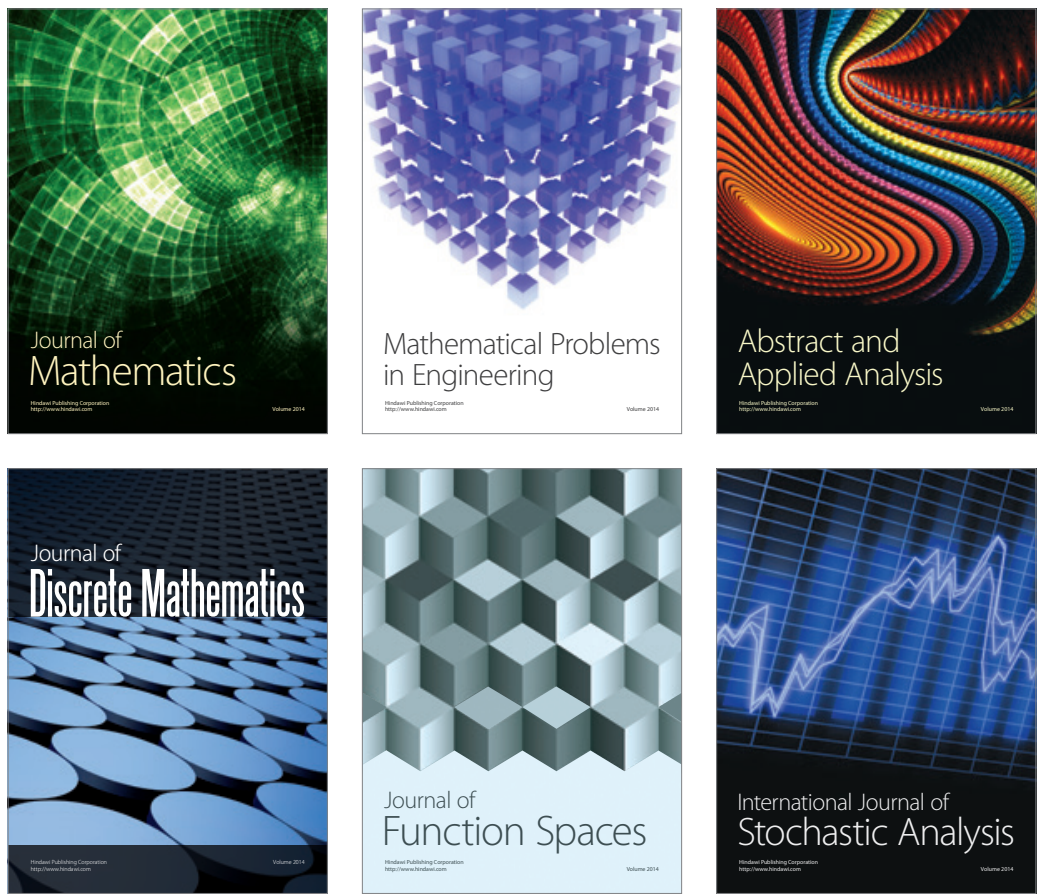

Journal of

Function Spaces

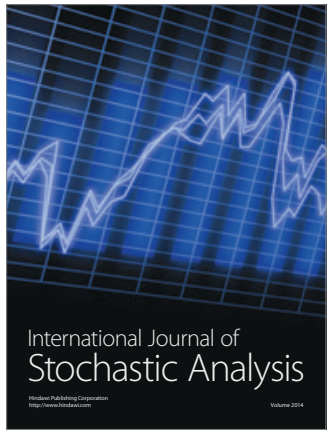

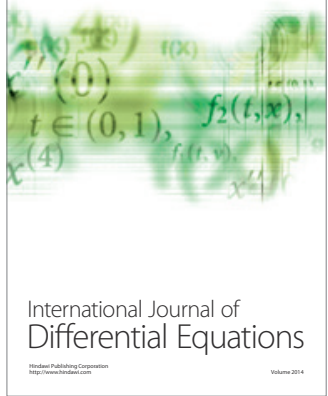
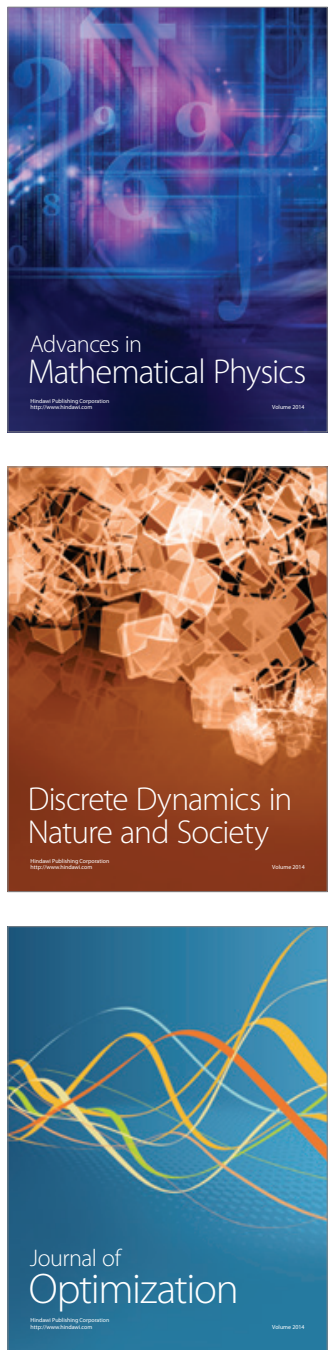